\title{
Keep your eyes on the ball: smooth pursuit eye movements enhance prediction of visual motion
}

\author{
Miriam Spering, ${ }^{1,2} *$ Alexander C. Schütz, ${ }^{1 *}$ Doris I. Braun, ${ }^{1}$ and Karl R. Gegenfurtner ${ }^{1}$ \\ ${ }^{1}$ Department of Psychology, Experimental Psychology, Justus-Liebig University, Giessen, Germany; and ${ }^{2}$ Department \\ of Ophthalmology and Visual Sciences, University of British Columbia, Vancouver, Canada
}

Submitted 13 April 2010; accepted in final form 31 January 2011

\begin{abstract}
Spering M, Schütz AC, Braun DI, Gegenfurtner KR. Keep your eyes on the ball: smooth pursuit eye movements enhance prediction of visual motion. J Neurophysiol 105: 1756-1767, 2011. First published February 2, 2011; doi:10.1152/jn.00344.2010.-Success of motor behavior often depends on the ability to predict the path of moving objects. Here we asked whether tracking a visual object with smooth pursuit eye movements helps to predict its motion direction. We developed a paradigm, "eye soccer," in which observers had to either track or fixate a visual target (ball) and judge whether it would have hit or missed a stationary vertical line segment (goal). Ball and goal were presented briefly for $100-500 \mathrm{~ms}$ and disappeared from the screen together before the perceptual judgment was prompted. In pursuit conditions, the ball moved towards the goal; in fixation conditions, the goal moved towards the stationary ball, resulting in similar retinal stimulation during pursuit and fixation. We also tested the condition in which the goal was fixated and the ball moved. Motion direction prediction was significantly better in pursuit than in fixation trials, regardless of whether ball or goal served as fixation target. In both fixation and pursuit trials, prediction performance was better when eye movements were accurate. Performance also increased with shorter ball-goal distance and longer presentation duration. A longer trajectory did not affect performance. During pursuit, an efference copy signal might provide additional motion information, leading to the advantage in motion prediction.
\end{abstract}

motion perception; direction prediction; fixation; efference copy

IN BALL SPORTS, ATHLETES ARE advised to keep their eyes on the ball to hit or catch it reliably. Professional athletes are often claimed to have better (i.e., faster, more precise) eye movements, and many studies have shown that humans track moving objects naturally in the context of performing a motor task such as playing baseball or driving a car [for an overview, see Land and Tatler (2009)]. On the other hand, it has been shown in the laboratory that the execution of pursuit eye movements can cause misperceptions of stationary and moving objects, an early observation that dates back to the $19^{\text {th }}$ century (Aubert 1887; von Fleischl 1882). Previous studies with real-world tasks described eye movement behavior associated with everyday motor activities but did not test whether eye movements improve perceptual performance, although this is frequently implied (e.g., Land, 2006). Here we experimentally addressed the question whether tracking a visual object with smooth pursuit eye movements improves the perception of its motion direction. Specifically, we designed a task, "eye soccer," that required observers to extrapolate the direction of a linearly

\footnotetext{
* M. Spering and A. C. Schütz contributed equally to this work.

Address for reprint requests and other correspondence: M. Spering, Dept. of Ophthalmology \& Visual Sciences, Univ. of British Columbia, Vancouver, Canada (e-mail: mspering@interchange.ubc.ca).
}

moving object and to predict whether this object (ball) would hit or miss a line segment (goal). We define motion prediction as the ability to anticipate a future event related to the moving object.

\section{Effects of Eye Movements on Motion Perception}

Many findings imply that eye movements are beneficial for tasks involving motion extrapolation. In ball sports, athletes use a combination of saccadic and smooth pursuit eye movements to track a moving ball, for instance, in baseball (Bahill and LaRitz 1984), basketball (Ripoll et al. 1986), cricket (Land and McLeod 2000), squash (McKinney et al. 2008), volleyball (Lee 2010), and even in table tennis (Land and Furneaux 1997), where ball movement time is very short. Pursuing the ball increases an observer's dynamic visual acuity and therefore enables the use of cues, such as the ball's spin, as a source of information on the ball's movement trajectory (Bahill et al. 2006). Similarly, when observers in the laboratory were asked to intercept the trajectory of a moving object with their hand or finger or to hit a moving object, they smoothly tracked the object until the moment of interception without instruction to do so (Brenner and Smeets 2009, 2011; Mrotek and Soechting 2007a; Soechting et al. 2009).

Whereas these studies suggest that smooth pursuit eye movements might improve motion prediction, pursuit also comes at a cost for motion perception in general. Pursuit can lead to misperceptions of the direction and speed of moving objects (Aubert 1887; Festinger et al. 1976; Filehne 1922; Freeman and Banks 1998; Haarmeier and Thier 1998; Morvan and Wexler 2009; Souman et al. 2005; von Fleischl 1882; Wertheim and Van Gelder 1990). Tracking a moving object with smooth pursuit eye movements produces a motion signal on the retina in the opposite direction to the pursuit object, induced by the relative motion of untracked objects in the background. Since we generally perceive stationary objects as stationary and moving objects as moving, even during pursuit, this eye-movement-induced retinal motion signal must be cancelled somewhere in the visuomotor processing stream to maintain perceptual stability. The cancellation process has to take into account that normal pursuit usually has a velocity gain of $<1$ (i.e., the eye lags behind the target). This difference between eye and target velocity (or internal and external signal) is referred to as retinal slip. Von Helmholtz (1910/ 1062) and von Holst and Mittelstaedt (1950) proposed that a compensation of eye-movement-induced motion signals might be achieved through a comparison of an external (retinal) motion signal with an internal (extraretinal) reference signal, 
informing the visual system about pursuit eye velocity so that retinal image motion can be interpreted and estimated.

Two key areas for visual motion processing in the primate cortex, the middle temporal area MT and the middle superior temporal area MST, might contribute to the interpretation of retinal image motion during pursuit in different ways. Recent neurophysiological studies in monkeys showed that MT activity was mostly correlated with target motion on the retina, whereas responses of some MST neurons were correlated with target motion on the screen, i.e., relative to the head and independent of the pursuit response (Chukoskie and Movshon 2009; Inaba et al. 2007). The finding that some MST neurons veridically encode retinal image motion during pursuit indicates that neurons in higher cortical visual areas compensate for eye-movement-induced motion signals during pursuit (see also Bradley et al. 1996; Dicke et al. 2008; Shenoy et al. 1999, Thier et al. 2001).

Even though the notion of a system that compensates for movement-induced motion signals is well established and its neural source identified, compensation during pursuit eye movements is usually imperfect. Perceptual discrepancies between fixation and pursuit arise for 1) stationary objects, 2) objects moving along with the pursuit target, and 3) objects moving perpendicular to the pursuit target. 1) In the Filehne illusion, a briefly presented stationary object appears to move in the direction opposite to the pursuit eye movement (Filehne 1922; Freeman and Banks 1998; Haarmeier and Thier 1998). 2) In the Aubert-Fleischl phenomenon, a visual object appears to move slower when it is smoothly tracked than when the observer views it during fixation (Aubert 1887; Turano and Heidenreich 1999; von Fleischl 1882; Wertheim and Van Gelder 1990). It has been reported that this difference in perceived speed does not affect discrimination accuracy in a velocity-matching task (Gibson et al. 1957). 3) Objects that move perpendicularly (Souman et al. 2005) or diagonally (Festinger et al. 1976; Morvan and Wexler 2009) relative to the pursuit trajectory are perceived to move at an angle rotated further away from the pursuit target.

To summarize, on the one hand, humans seem to track moving objects naturally when performing a motor task, presumably to enable better motion prediction and to thereby aid motor planning. On the other hand, the execution of smooth pursuit can alter the perception of stationary and moving objects in laboratory tasks. Are smooth pursuit eye movements beneficial or detrimental for object motion prediction when onset, direction, angle, and duration of the object motion are uncertain?

\section{METHODS}

We compared the ability to predict a moving object's motion direction by extrapolating its trajectory during pursuit and fixation in three experiments (methods for experiment 1 described here; for experiments 2 and 3, see RESULTS). We introduce a novel paradigm, "eye soccer," in which observers had to judge whether a briefly presented object (the "ball") would hit or miss a line segment (the "goal"), while fixating or smoothly pursuing the ball. This task requires the ability to predict a visual motion trajectory, because both ball and goal were blanked before the hit or miss event.

\section{Observers}

Observers (mean age $24.2 \pm 1.8 \mathrm{yr}$ ) were undergraduate students from Giessen University, Germany, and participated with informed consent. All observers were unaware of the purpose of the experiment and had normal or corrected-to-normal visual acuity. Experiments were in accordance with the principles of the Declaration of Helsinki and approved by the local ethics committee.

\section{Visual Stimuli and Apparatus}

Visual stimuli were presented on a " 21 " CRT monitor at a refresh rate of $100 \mathrm{~Hz}$, set to a spatial resolution of $1280(\mathrm{H}) \times 1024(\mathrm{~V})$ pixels. Observers viewed stimuli binocularly from a distance of $47 \mathrm{~cm}$ with their head stabilized by a chinrest. The ball (white or red Gaussian dot, $\mathrm{SD}=0.15^{\circ}$ ) and goal (vertical white line segment, $3^{\circ}$ long, $0.15^{\circ}$ wide) were presented on a uniform black background. To prohibit the use of external reference frames as indicators of target position, experiments took place in a dark, windowless and completely light-shielded room that had black walls and a black curtain at the bottom edge of the door. All light sources (e.g., from computer mouse or power outlet switches) were covered with black tape. The monitor frame was covered with nonreflecting black cardboard and fabric. To block residual light from the monitor itself, two neutraldensity filters (LEE Filters, Burbank, CA) were mounted in front of the display. Through the filters, the black background had a luminance of $<0.001 \mathrm{~cd} / \mathrm{m}^{2}$; white and red pixels had a luminance of 1 and 0.11 $\mathrm{cd} / \mathrm{m}^{2}$, respectively. As a result of these measures, observers could not see any visual references such as the monitor frame.

\section{Experimental Procedure and Design}

Figure 1 shows the sequence of events in individual trials. We compared direction prediction performance during fixation and pursuit. A given trial could either be a fixation or a pursuit trial, randomly interleaved in a block of trials. A white fixation spot shown at the beginning of the trial (Fig. 1, la) indicated pursuit, a red fixation spot (Fig. 1, $1 b$ ) indicated fixation. In both types of trials, the ball served as the eye movement target. In fixation trials, the ball remained stationary and had to be fixated while the goal moved towards the fixation position (instruction to fixate the ball). In pursuit trials, the ball moved towards the stationary goal and had to be pursued (instruction to pursue the ball). The initial horizontal and vertical position of the fixation spot was varied from trial to trial within a range of $3.5^{\circ}$ around the center of the monitor. The onset of stimulus motion, either of the goal in fixation trials or of the ball in pursuit trials, was initiated with a button press by the observer. In the fixation condition, observers were instructed to maintain fixation on the initial fixation spot. In the pursuit condition, the initial fixation spot became the pursuit target when it started to move. To keep retinal stimulation as similar as possible in both conditions, the ball turned white in the fixation condition (Fig. 1, 2b) once the goal started to move. Importantly, the ball and goal disappeared simultaneously before a judgment was made. At the end of each trial, observers were asked to press an assigned button to indicate whether the target would have hit or missed the goal, if motion had continued. No performance feedback was given.

Ball or goal speed was constant at $10 \%$ s. Hit positions were on the goal, at $0.25^{\circ}$ from the goal endpoints towards the goal center, and miss positions were outside the goal, at $0.25^{\circ}$ from the goal endpoints (see Fig. 2 for an example). Task difficulty was manipulated through variation of presentation duration $(100,300$, or $500 \mathrm{~ms})$ and ball-goal distance upon disappearance $\left(3\right.$ and $\left.6^{\circ}\right)$. To make ball or goal motion less predictable, trajectory direction and angles were varied. The target (ball or goal) moved either to the left or right and either along the horizontal meridian $\left(0^{\circ}\right.$ angle) or diagonally up $\left(+15^{\circ}\right.$ from horizontal) or down $\left(-15^{\circ}\right.$; see Fig. 1 , bottom left $)$. Conditions and 


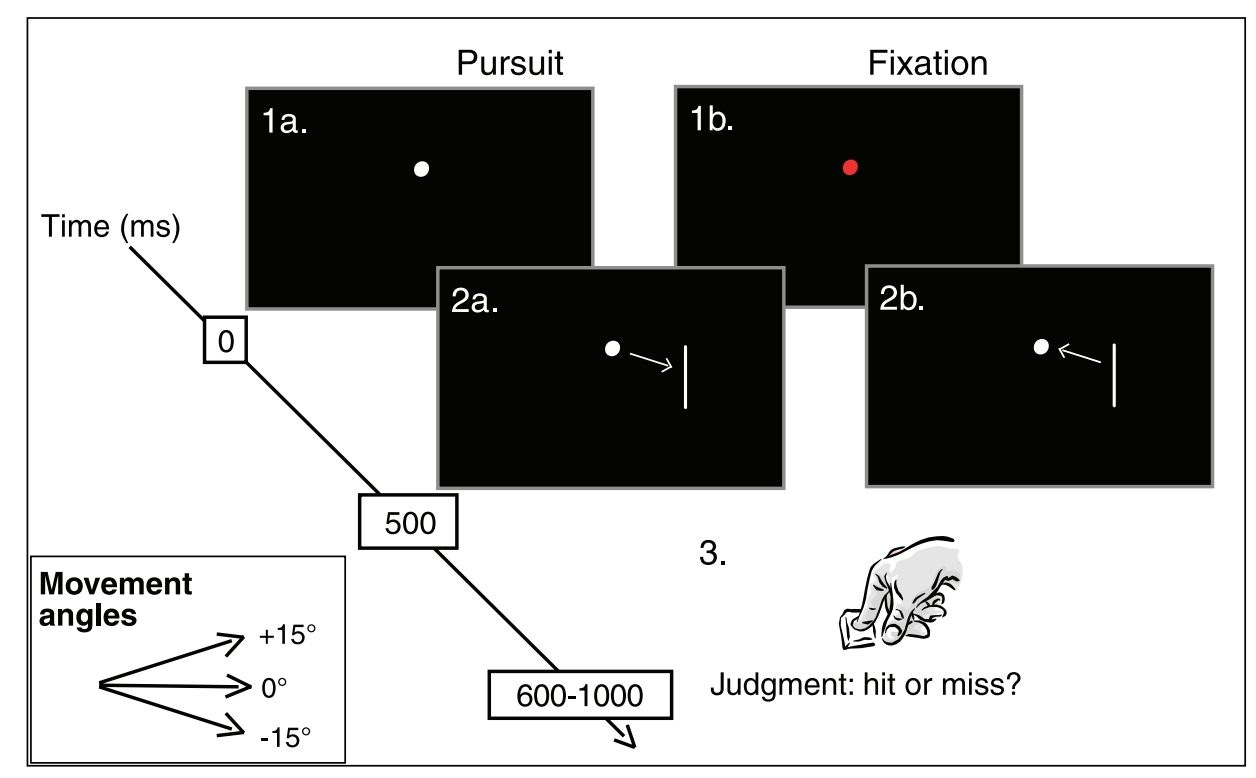

Fig. 1. Trial sequence for a "hit" trial in eye soccer. 1: initial fixation and eye-tracker drift correction (la: pursuit trial: ball white; $1 b$ : fixation trial: ball red). $2 a$ : pursuit: step-ramp motion of ball towards goal; $2 b$ : fixation: motion of goal towards stationary ball; both balls white. 3: disappearance of ball and goal before ball reached goal; perceptual judgment. Possible ball or goal motion angles $\left(0^{\circ}\right.$ and $\left.\pm 15^{\circ}\right)$ are illustrated in bottom left. movement direction and angles were randomly interleaved in each block of trials.

\section{Eye Movement Recordings and Analysis}

Eye position was monitored with a head-mounted, video-based eye tracker (EyeLink II; SR Research, Osgoode, Ontario, Canada) and sampled at $250 \mathrm{~Hz}$. Eye velocity was obtained by digital differentiation of eye position signals over time and filtered using a low-pass, second-order Butterworth filter with a cutoff at $40 \mathrm{~Hz}$. Horizontal and vertical saccades were removed from the unfiltered traces and replaced by linear interpolation between saccade onset and offset. Saccade onset and offset detection was based on the third derivative of eye position over time (jerk), obtained by differentiating unfiltered eye acceleration. Four consecutive samples had to exceed a fixed criterion of $95,000 \% \mathrm{~s}^{3}$ to be counted as saccade samples. Pursuit onset was detected using a piecewise linear fit to the filtered velocity trace.

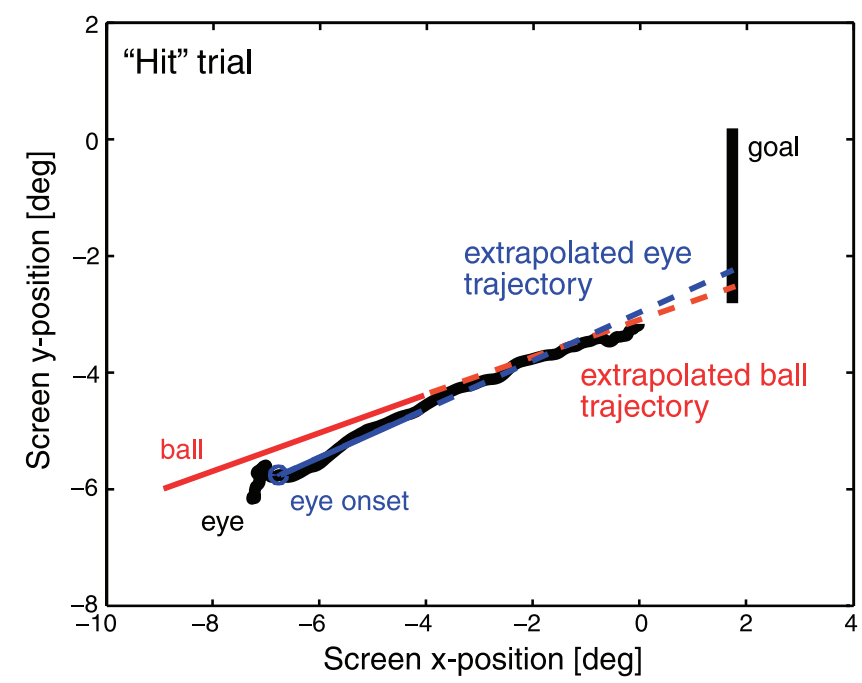

Fig. 2. Representative "hit" trial from one observer in experiment 1. 2-D eye position (black) and ball position (red) relative to goal position (vertical black line). Solid red line denotes the visible ball trajectory on the screen, and dashed red line denotes the extrapolated ball position between ball offset and ball end position if the ball had hit the goal. The solid blue line denotes the regression fit to eye position, and the dashed blue line denotes the extrapolated eye position.
All traces were visually inspected, and traces with eye blinks or undetected saccades or pursuit onsets were excluded from analysis $(\sim 0.2 \%$ of all trials in any experiment).

To make sure that pursuit was elicited in pursuit trials and that fixation was maintained in fixation trials, we included pursuit trials in further analyses only if 1) a pursuit onset was found and 2) the mean 2 -D position error in the interval between eye movement onset and $300 \mathrm{~ms}$ after onset was $<2^{\circ}$. Similarly, fixation trials were only included if 1 ) no pursuit onset was found, and 2) the eye remained within a $2^{\circ}$ circle of initial fixation position. Based on these criteria, $581(10.1 \%)$ out of a total of 5,760 trials were excluded in experiment 1 , resulting in 5,179 remaining trials for analysis.

We calculated retinal slip in pursuit and fixation trials. For pursuit, retinal slip was defined as the difference in velocity between the ball $(10 \%)$ and the eye from ball motion onset to offset. For fixation, retinal slip was the velocity difference between the ball $(0 \%)$ and the eye from goal motion onset to offset or simply the mean eye velocity during this time interval. We also calculated pursuit steady-state gain during the closed-loop phase, 200-400 ms after pursuit onset, when the eye velocity can be expected to match the target velocity optimally.

\section{Analysis of Perceptual and Pursuit Judgments}

Observers' perceptual performance was quantified using the sensitivity measure $d$ prime $\left(\mathrm{d}^{\prime}\right)$. Generally, perceptual responses can be classified into "hits" (in eye soccer: judgment "goal," target goal), "misses" (judgment "miss," target goal), "correct rejections" (judgment "miss," target missed), and "false alarms" (judgment "goal," target missed). The value of $\mathrm{d}^{\prime}$ is an index of how well an event (a goal or a miss) can be detected. It is generally believed to be uncontaminated by response bias (such as responding "goal" more often as the number of goal trials increases). It is defined as

$$
\mathrm{d}^{\prime}=z(H)-z(F)
$$

where $z(H)$ and $z(F)$ are experimentally determined $z$-transformed hit and false alarm rates, respectively. We also report the proportion of correct $(\mathrm{PC})$ trials as

$$
\mathrm{PC}=\left(n_{\text {Hits }}+n_{C R}\right) / n_{\text {Total }}
$$

where $n_{H i t s}$ and $n_{C R}$ refer to numbers of "hits" and "correct rejections," respectively. The PC is informative with regard to the source of potential differences between $\mathrm{d}^{\prime}$ in different conditions: it can 
reveal whether these are due to differences in hit rates (PC should parallel differences in $\mathrm{d}^{\prime}$ ) or false alarm rates (PC should not reflect differences in $\mathrm{d}^{\prime}$ ).

We further analyzed $\mathrm{d}^{\prime}$ for pursuit responses: linear regression lines of $200 \mathrm{~ms}$ in length were fitted to the 2-D eye position traces in the time interval 100-450 ms after pursuit onset (Fig. 2). The regression windows were moved in 50-ms steps along the eye position trace and linearly extrapolated to obtain the intersection with the goal line segment, yielding four analysis intervals starting at 100, 150,200, and $250 \mathrm{~ms}$ after pursuit onset. Based on the intersection point, the pursuit response was classified as hit, miss, correct rejection, or false alarm, and $\mathrm{d}^{\prime}$ was calculated. For each analysis interval, we calculated the fraction of variance that was unexplained by the regression, defined as

$$
\mathrm{FVU}=\frac{\sum_{i=1}^{N}\left(y_{i}-\hat{y}_{i}\right)^{2}}{\sum_{i=1}^{N}\left(y_{i}-\bar{y}_{i}\right)^{2}}
$$

where $i$ is a counting variable from 1 to the number of all regression points, $y$ are observed values, $\hat{y}$ are values predicted by the regression, and $\bar{y}$ is the mean of observed values. The numerator indicates the sum of squared deviations of the regression from observed values; the denominator indicates the sum of squared deviations of the regression from the mean of observed values. Note that fraction of variance that is unexplained (FVU) corresponds to $1-R^{2}$ and ranges from 0 (perfect fit) to 1 (fit only explains mean across all time intervals).

To analyze the agreement between perceptual judgments and pursuit responses, we calculated the proportion of trials with same judgments in perception and pursuit $\left(\mathrm{P}_{\text {Same }}\right)$ and compared this to the proportion of same judgments that is to be expected if responses are random (see also Gegenfurtner and Franz 2007; Stone and Krauzlis 2003), defined as

$$
\mathrm{P}_{\text {Chance }}=\mathrm{P}_{\text {Prec }} * \mathrm{P}_{\text {Purs }}+\left[\left(1-\mathrm{P}_{\text {Perc }}\right) *\left(1-\mathrm{P}_{\text {Purs }}\right)\right]
$$

\section{RESULTS}

\section{Experiment 1}

Perceptual motion prediction is better during pursuit than during fixation. In experiment 1, we compared motion prediction during pursuit and fixation and varied presentation duration and ball-goal distance in five observers. Pursuit improved motion prediction: Observers were better in predicting the direction of a moving ball when they tracked it with their eyes (i.e., higher $\mathrm{d}^{\prime}$ values in Fig. $3 A$ and higher proportion correct in Fig. $3 C$ ) than when they fixated the ball while the goal was approaching it.

We compared perceptual performance $\left(\mathrm{d}^{\prime}\right)$ during pursuit and fixation using three-way repeated-measures ANOVA with factors eye condition, duration, and distance. Three main effects were obtained. Perceptual performance was better during pursuit than during fixation under all experimental conditions $[F(1,4)=8.0, P=0.048$; compare black symbols for pursuit with red symbols for fixation in Fig. 3, $A$ and $C$ ]. As expected, performance increased with presentation duration $[F(2,8)=9.4, P=0.01]$. Performance was also higher when the distance between ball and goal was smaller $[F(1,4)=24.5$, $P=0.01$; compare circles for small distance to squares for large distance in Fig. 3A]. There were no significant interactions. The main effects of eye condition and duration were reflected in the percentage of correct responses (Fig. 3C); the effect of distance was not. This finding indicates that the overall difference in $\mathrm{d}^{\prime}$ between fixation and pursuit and the effect of presentation duration was mostly due to the proportion of correct trials, whereas the effect of distance was
A

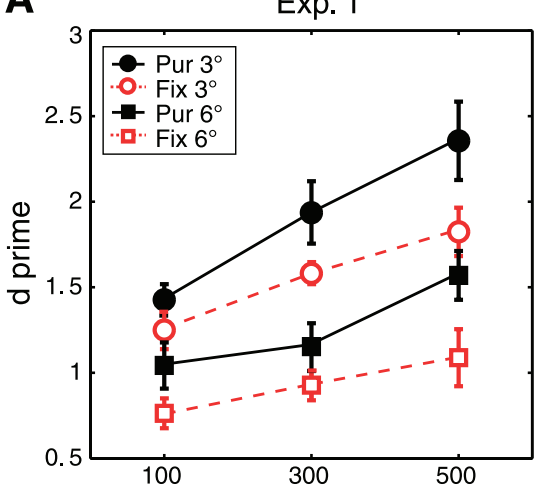

C

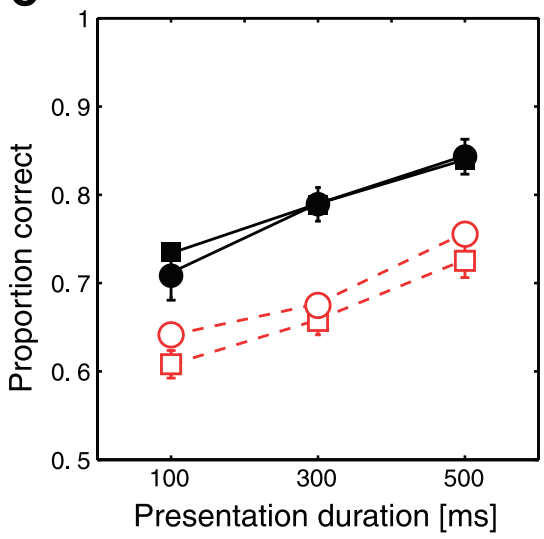

B

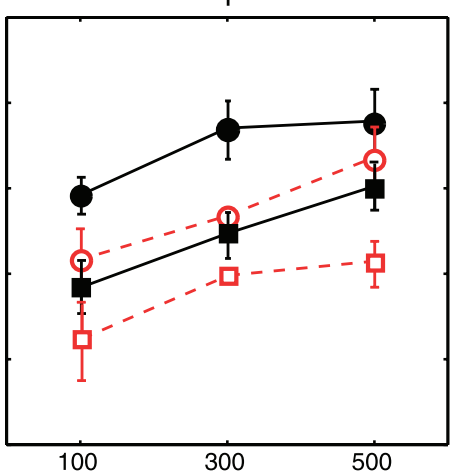

D

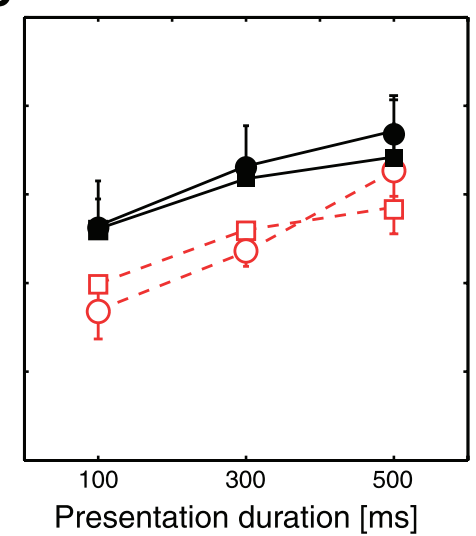

Fig. 3. Comparison of perceptual results ( $\mathrm{d}^{\prime}$ and PC) between pursuit (black solid symbols and lines) and fixation (red open symbols and dashed lines) for 2 ball-goal distances and 3 presentation durations. $A$ and $C$ : results for experiment 1 ; fixation on ball with goal moving in fixation condition (5 observers). $B$ and $D$ : experiment 2 ; fixation on goal with ball moving in fixation condition (4 observers). Pursuit conditions were identical in experiments 1 and 2. $C$ and $D$ : proportion correct. Data are means $\pm \mathrm{SE}$. 
due to the false-alarm rate (higher in trials with larger distance).

Effect of motion angle. The results presented so far were averaged across motion directions (left, right) and angles $\left(0^{\circ}, \pm 15^{\circ}\right)$. Overall perceptual performance $\left(\mathrm{d}^{\prime}\right)$ was not significantly affected by motion direction $[F(1,4)=0.25, P=$ $0.64]$ but depended significantly on motion angle $[F(2,8)=$ $7.49, P=0.01]$ with better overall performance in conditions with horizontal $\left(0^{\circ}\right.$; right: $\mathrm{M}=1.79 \pm 0.63$, left: $\left.2.18 \pm 0.81\right)$ than with nonhorizontal, diagonal motion angle $\left( \pm 15^{\circ}\right.$; right up: $\mathrm{M}=1.21 \pm 0.24$, right down: $1.16 \pm 0.26$, left up: $1.17 \pm$ 0.17 , left down: $1.19 \pm 0.31)$. Differences between the two nonhorizontal motion angles were not significant, as indicated by Bonferroni-corrected post hoc $t$-tests [right up vs. right down: $t(8)=0.26, P=87$; left up vs. left down: $t(8)=-0.09$, $P=0.95]$. The finding that overall performance was better along the horizontal axis than along nonhorizontal, diagonal axes reflects the well-known oblique effect (Ball and Sekuler 1982; Furmanski and Engel 2000). However, regardless of motion angle, we found the same three main effects as reported above: perceptual motion prediction was better in pursuit than in fixation trials [horizontal: $F(1,4)=14.9, P=0.02$; nonhorizontal: $F(1,4)=8.4, P=0.03$ ], better for short than for long distances [horizontal: $F(1,4)=49.2, P=0.002$; nonhorizontal: $F(1,4)=13.3, P=0.02$ ], and better for longer than for shorter presentation durations [horizontal: $F(2,8)=9.6, P=$ 0.008; nonhorizontal: $F(2,8)=7.3, P=0.02]$. For the following analyses, we averaged across motion directions and angles.

Can we assume similarity of retinal motion signals during pursuit and fixation? The rationale for a fixation condition in which the ball was stationary and the goal moved towards the ball was to ensure similarity between pursuit and fixation conditions in terms of retinal image motion with respect to both ball and goal. However, similarity can only be assumed under ideal circumstances, in which eye velocity gain during pursuit is 1 and eye velocity during fixation is 0 . Usually, pursuit gain is $<1$ and fixation is not stable due to small eye movements such as microsaccades and drift. Figure 4 shows mean eye position and velocity traces relative to pursuit onset. Velocity gain was $<1$ and strongly depended on presentation duration (see also Table 1). Imprecise pursuit and fixation are only a concern here if they affect perceptual performance to an extent that they explain the performance difference between pursuit and fixation trials.

First, to assess the precision of pursuit and fixation, we analyzed retinal slip in pursuit and fixation trials (see METHODS), reported in Table 1. A lower retinal slip implies higher precision in pursuit and fixation and presumably also better motion perception. Retinal slip was lower during fixation than during pursuit $[F(1,4)=623.7, P<0.0001]$, indicating that our main result, better perceptual performance during pursuit than during fixation, is not due to inaccurate eye movements during fixation. In both pursuit and fixation trials, retinal slip was significantly affected by presentation duration and decreased with increasing duration [fixation: $F(2,8)=23.3, P<0.0001$; pursuit: $F(2,8)=117.9, P<0.0001]$. In fixation trials, retinal slip did not vary significantly with distance $[F(1,4)=1.01$, $P=0.373$ ] whereas pursuit quality improved with longer distance $[F(1,4)=36.3, P=0.004]$. It is known that stationary backgrounds reduce initial pursuit acceleration (Keller and Khan 1986) and steady-state gain (Collewijn and Tamminga 1984). The stationary goal line segment in "eye soccer" might have had a similar effect, particularly in the short-distance conditions.

We next asked whether eye movement precision could explain the observed performance difference in motion direction prediction between pursuit and fixation trials. In Fig. 5, we show a comparison between perceptual performance in trials with best (top 25\%) and worst (bottom 25\%) eye movement precision for fixation (Fig. 5A) and pursuit (Fig. 5B), respectively. Retinal slip in fixation trials was $\mathrm{M}=1.6 \pm 0.25 \% \mathrm{~s}$ in trials with good fixation (Fig. 5A, solid red symbols) and $\mathrm{M}=2.5 \pm 0.17 \%$ s (Fig. $5 A$, open red symbols) in trials with bad fixation; retinal slip in pursuit trials was $\mathrm{M}=5.0 \pm 0.23^{\circ} \mathrm{s}$ in trials with good pursuit (Fig. $5 B$, solid black symbols) and $\mathrm{M}=7.1 \pm 0.34 \%$ (Fig. $5 B$, open black symbols) in trials with bad pursuit. Note that retinal slip in pursuit trials was calculated from stimulus onset to stimulus offset for comparison with fixation trials, and therefore included the latency and open-loop phases of pursuit during which eyetarget velocity matching is usually zero or low, respectively. For comparison, we also report pursuit steady-state gain calculated during the time interval $200-400 \mathrm{~ms}$ after pursuit onset (Table 1). Although steady-state gain was low $(<0.5)$
Fig. 4. Mean eye position $(A)$ and velocity traces $(B)$ relative to pursuit onset in experiment 1 for five observers. Colors denote presentation durations. Vertical black dashed lines denote beginning and end of fitting intervals (see METHODS and Fig. 6).
A

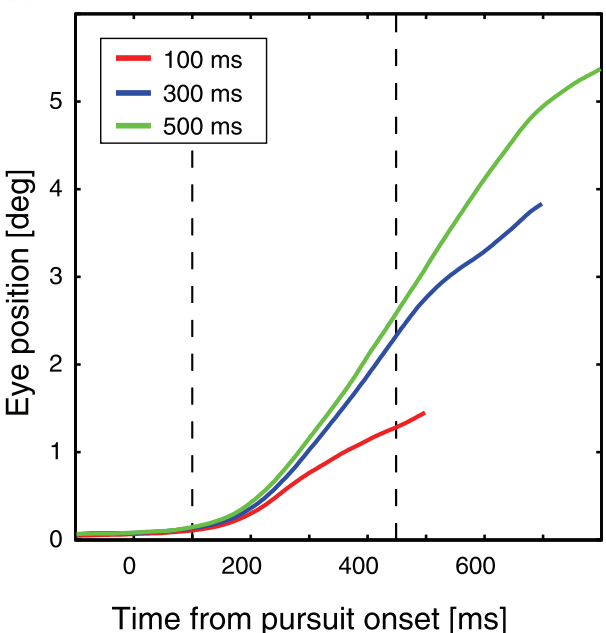

Time from pursuit onset [ms]
B

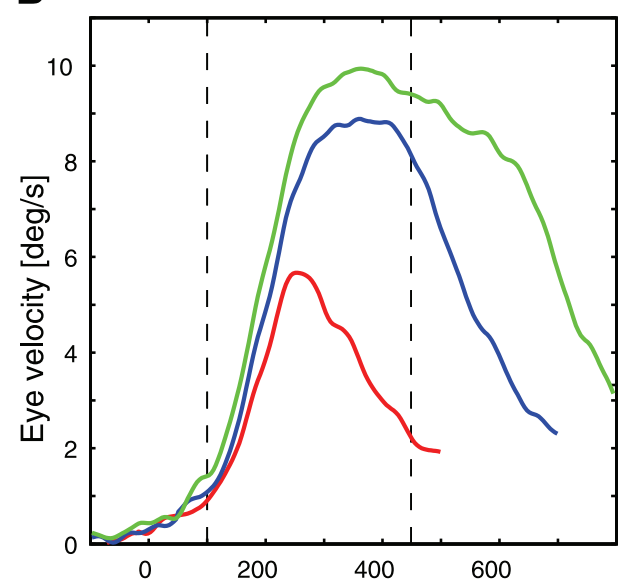

Time from pursuit onset [ms] 
Table 1. Retinal slip in fixation and pursuit trials and pursuit steady-state gain for six conditions (distance and duration) in experiment 1

\begin{tabular}{|c|c|c|c|c|c|c|}
\hline $3^{\circ}, 300 \mathrm{~ms}$ & 2.09 & 0.24 & 7.03 & 0.35 & 0.62 & 0.11 \\
\hline $3^{\circ}, 500 \mathrm{~ms}$ & 2.05 & 0.26 & 6.03 & 0.43 & 0.82 & 0.12 \\
\hline $6^{\circ}, 100 \mathrm{~ms}$ & 2.36 & 0.23 & 7.99 & 0.31 & 0.47 & 0.11 \\
\hline $6^{\circ}, 300 \mathrm{~ms}$ & 2.19 & 0.17 & 6.56 & 0.51 & 0.83 & 0.06 \\
\hline
\end{tabular}

for the short presentation duration, it increased with presentation duration to $>0.9$ for the 500 -ms presentation duration (see also Fig. 4). The high retinal slip for pursuit was therefore likely due to the long analysis interval as well as to the short presentation duration.

For both fixation and pursuit, perceptual performance increased with increasing eye movement precision. However, even with as close to optimal fixation as achieved by our observers, perceptual performance in those trials was generally worse than performance in trials with good pursuit (compare solid red symbols in Fig. 5A and solid black symbols in Fig. $5 B$ ). Moreover, performance in trials with good fixation was only as good as performance in trials with bad pursuit (compare solid red symbols in Fig. $5 \mathrm{~A}$ to and open black symbols in Fig. $5 B$ ). It follows that imprecise fixation and pursuit compromised perceptual performance but could not explain the perceptual performance difference between pursuit and fixation trials. Moreover, the analysis of retinal slip also demonstrated that the retinal image was not the same in fixation and pursuit trials.

Does better pursuit lead to better perceptual performance? We next asked whether the perceptual performance difference between pursuit and fixation was reflected in the pursuit detection performance (pursuit d', METHODS). Does better pursuit lead to better perceptual performance? The analysis in Fig. 5 showed that perceptual performance was on average better in trials with high pursuit precision. To compare perceptual and pursuit performance more closely, we analyzed the temporal development of pursuit d' (METHODS; Fig. 2). Pursuit performance was generally higher for longer presentation durations (300 and $500 \mathrm{~ms}$ ) than for the short one and increased over time (Fig. 6, $A$ and $B$ ). When the stimuli were presented for only $100 \mathrm{~ms}$, pursuit performance decreased to chance level for the fourth fitting interval. This finding is not unexpected. In line with Barnes and Collins (2008), we found that a sampling period of $100 \mathrm{~ms}$ was sufficient to produce a reliable smooth pursuit response in a substantial number of all trials with pursuit instruction (73\%) but still in fewer trials than for longer presentation durations (300 ms: 91\%, $500 \mathrm{~ms}:$ 93\%). The decrease in pursuit performance at the shortest presentation duration during the last analysis interval might also reflect the small amount of pursuit executed when targets disappeared before the onset of pursuit (pursuit latency: $\mathrm{M}=150.8, \mathrm{SD}=$ 2.2; interestingly, latency was similar for longer presentation durations, $300 \mathrm{~ms}: \mathrm{M}=150.2 \pm 4.1,500 \mathrm{~ms}: \mathrm{M}=149.9 \pm$ $4.2)$, and when the analysis interval started at $\sim 350 \mathrm{~ms}(\sim 150$ ms latency $+200 \mathrm{~ms}$ until start of analysis interval) after the targets disappeared. Moreover, in the 100-ms condition, pursuit gain started to decrease rapidly at $\sim 180 \mathrm{~ms}$ after pursuit onset (see Fig. 4), affecting the third (200-400 ms) and fourth $(250-450 \mathrm{~ms})$ fitting interval. The FVU by the individual regressions was $<0.43$ for all conditions and analysis intervals, indicating good fits. In correspondence with data in Fig. 6, the FVU was higher for the shortest presentation duration $(\mathrm{M}=$ $0.39 \pm 0.11$ and $0.34 \pm 0.08$ for short and long distance, respectively) than for the 300-ms $(M=0.29 \pm 0.06$ and $0.25 \pm 0.06)$ or the $500-\mathrm{ms}$ conditions $(\mathrm{M}=0.26 \pm 0.06$ and $0.24 \pm 0.05)$.

Pursuit $\mathrm{d}^{\prime}$ in Fig. 6, $A$ and $B$, represents the motion information that is available to the perceptual system for direction prediction from the pursuit eye movements alone. Is this information, when combined with the pure perceptual information obtained in fixation trials (in Fig. 3A, open symbols), sufficient to account for the perceptual performance benefit in pursuit trials (compare open with filled symbols in Fig. 3A)? When averaged across time, pursuit $\mathrm{d}^{\prime}$ is $\sim 0.6$ for the two
A

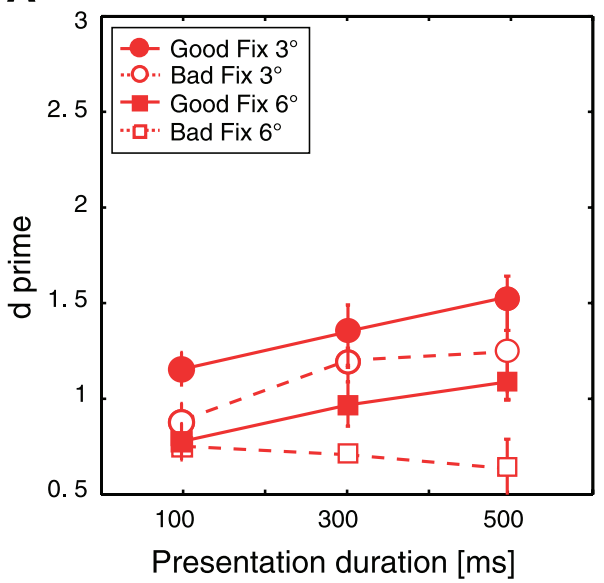

B

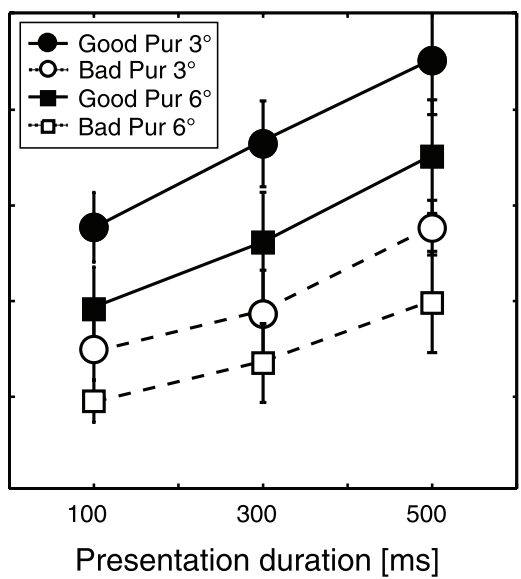

Fig. 5. Comparison of perceptual $\mathrm{d}^{\prime}$ between trials with good and bad eye movement precision in experiment 1. A: fixation trials; results under good fixation denoted by solid symbols and lines, and results under bad fixation denoted by open symbols and dashed lines. $B$ : pursuit trials with same format as in $A$. 
A

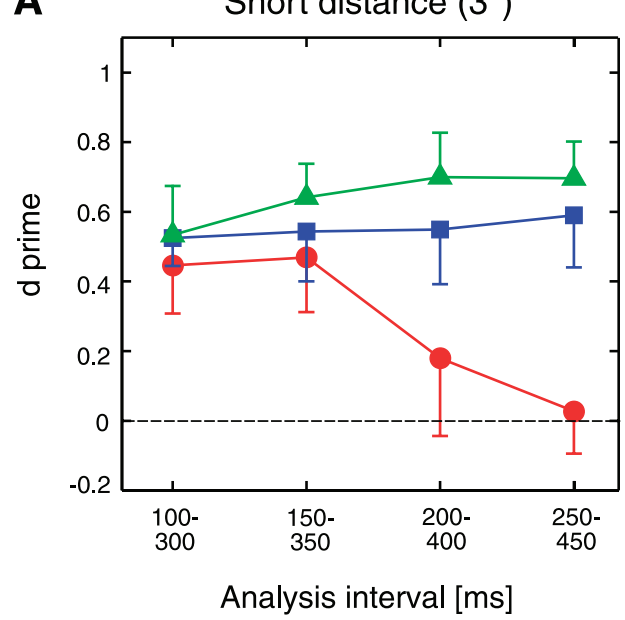

B

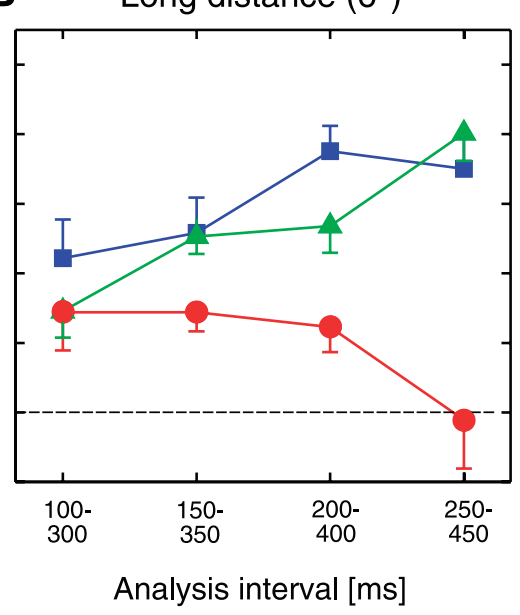

Fig. 6. Eye movement performance (pursuit d') yielded from extrapolated eye movement direction in experiment 1. A: pursuit $\mathrm{d}^{\prime}$ for short distance $\left(3^{\circ}\right)$ between ball and goal. Results are plotted for four 200-ms fitting intervals over a total time period from 100 to $450 \mathrm{~ms}$ after pursuit onset. Symbols denote presentation durations. Data are means $\pm \mathrm{SE}$. $B$ : pursuit $\mathrm{d}^{\prime}$ for long distance $\left(6^{\circ}\right)$, same format as in $A$. interval (200-400 ms; Fig. 7C). We compared mean $\mathrm{P}_{\text {Same }}$ and $\mathrm{P}_{\text {Chance }}$ values per subject for all analysis intervals in a paired-samples $t$-test and obtained a significant difference for the last interval [Fig. $7 C ; t(4)=3.3, P=0.01$ ] but not for the first two intervals (Fig. 7, $A$ and $B$ ).

The increase in $\mathrm{P}_{\text {Same }}$ values over time reflects a well-known property of the pursuit response: eye-target velocity matching is usually not optimal during the pursuit initiation phase, which lasts up to $\sim 150 \mathrm{~ms}$ after pursuit onset (Lisberger et al. 1987; Osborne et al. 2007). For a relatively arbitrary early pursuit response with respect to velocity matching, the agreement between perception and pursuit can only be at chance level but will increase as the fitting interval moves into the pursuit maintenance phase, where velocity matching is often close to perfect.

Generally, the finding that perceptual performance was reflected in the steady-state pursuit response has implications for the mechanism underlying the enhancement of motion prediction through pursuit. The better the pursuit (i.e., the closer its velocity matches target velocity), the more precise an internally generated motion signal (e.g., efference copy), which could potentially be used to improve perception (see DISCUSSION). (100-300 ms; Fig. 7A) to good agreement in the latest
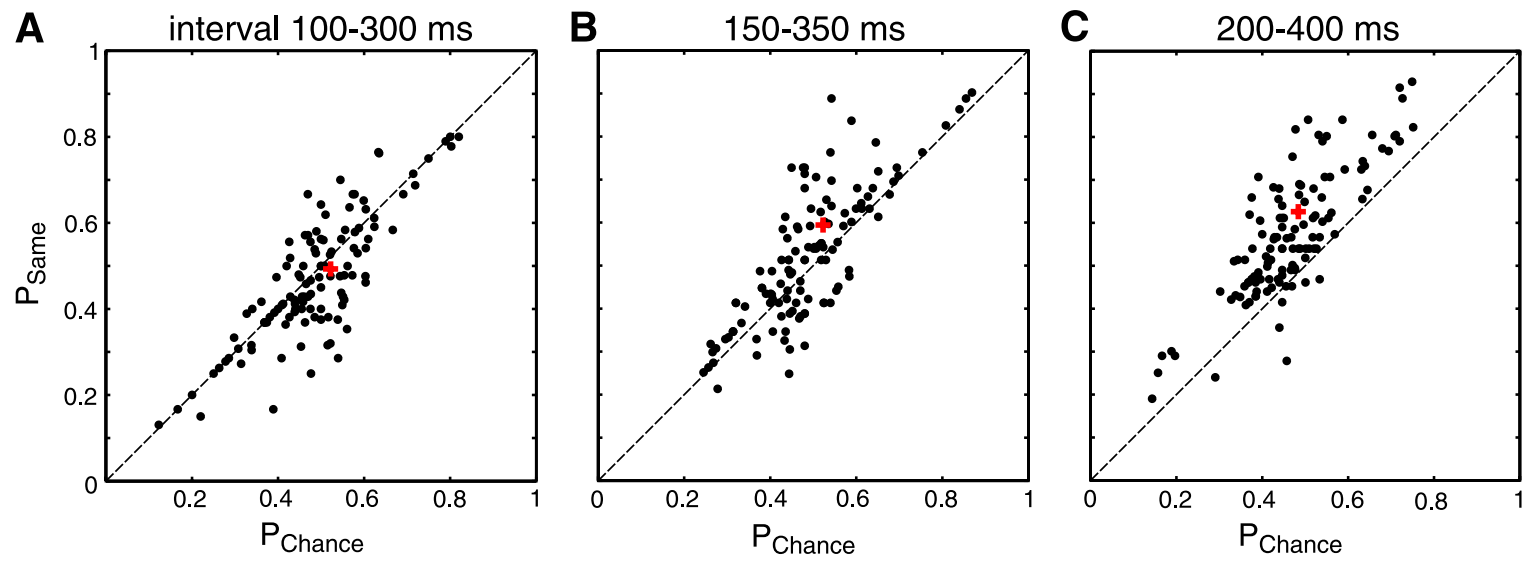

Fig. 7. Agreement between perceptual and pursuit responses across observers and conditions in experiment 1 plotted for different time intervals after pursuit onset. A: fitting interval 100-300 ms after pursuit onset. $B$ : interval $150-350 \mathrm{~ms}$. $C$ : interval 200-400 ms. Red cross marks mean values of judgments that were the same $\left(\mathrm{P}_{\mathrm{Same}}\right)$ and judgments that followed a random response pattern $\left(\mathrm{P}_{\text {Chance }}\right)$ across conditions. Diagonal dashed line marks boundary between agreement (points falling above the line) and chance performance (points falling below the line). 
Experiment 2: Pursuit Enhances Motion Prediction Irrespective of Retinal Image Motion

In experiment 1 , retinal motion information in pursuit and fixation conditions was similar. Observers were asked to fixate a stationary ball while the goal was moving towards it, and ball and goal were blanked simultaneously. However, in a more realistic situation observers might fixate on the goal, the "center of action," instead. In experiment 2, we tested whether results depended on fixation location by having four observers to fixate on a fixation spot in the goal center ("fixate the goal") while the ball was moving towards the goal. The pursuit condition was the same as in experiment 1 ("pursue the ball"). All other conditions were identical to experiment 1. Based on the criteria for eye movement quality (see METHODS), we excluded $166(5.4 \%)$ out of a total of 3,072 trials. As in experiment 1 , overall perceptual performance was better for horizontal $(\mathrm{M}=2.12 \pm 0.92)$ than for nonhorizontal motion angles $(\mathrm{M}=1.41 \pm 0.24)$, but not significantly $[F(1,3)=3.7, P=$ 0.15]. Regardless of motion angle, results (Fig. $3 B$ ) show that, in line with our previous findings, perceptual performance $\left(\mathrm{d}^{\prime}\right)$ was better in pursuit than in fixation trials $[F(1,3)=17.3, P=$ $0.03]$, better for short than for long distances $[F(1,3)=18.0$, $P=0.02]$, and better for longer than for shorter presentation durations $[F(2,6)=20.3, P=0.002]$. There were no significant interactions. To test if fixation performance differed in experiments 1 and 2 we calculated a three-way repeatedmeasures ANOVA with within-subject factors duration and distance and experiment as between-subject factor. Performance was better for short distances $[F(1,7)=26.01$, $P=0.001]$ and for long presentation durations $[F(2,14)=$ $6.04, P=0.01]$. There was no significant difference in performance between experiments 1 and $2[F(1,7)=2.75, P=0.14]$ and no significant interactions.

As in experiment 1, only the main effects of eye condition and duration were reflected in the proportion of correct responses (Fig. 3D). Findings in experiment 2 indicate that pursuit improves perceptual performance irrespective of the fixation position in the visual field, i.e., the corresponding retinal motion information.

\section{Experiment 3: Motion Prediction During Pursuit and Fixation Is Independent of Trajectory Length}

Presentation duration and trajectory length were covaried in experiments 1 and 2 . The relative importance of spatial and temporal information for the present task is therefore unclear. It has been shown previously (Whitaker et al. 2008) that thresholds for discriminating angular deviations in moving objects depend on presentation duration only and not on path length. This study used a different task, observers had to discriminate direction changes in moving objects, and did not take eye movements into account. In experiment 3 , we manipulated the duration of the motion path by varying stimulus speed, while keeping trajectory length constant. To travel the same distance, a stimulus with short presentation duration moved faster, and a stimulus with long presentation duration moved more slowly. As in experiments 1 and 2, presentation durations were 100, 300, and $500 \mathrm{~ms}$. The distance between ball and goal was constant at $6^{\circ}$, and trajectory length was 1,3 , or $5^{\circ}$. Accordingly, stimulus speeds varied between $2-30 \%$ s, depending on presentation duration and trajectory length. Seven observers participated. Results are based on a total of 10,945 trials (1,367 or $11.1 \%$ out of 12,312 trials were excluded).

As in experiment 1 , overall perceptual performance was significantly better for horizontal $(\mathrm{M}=1.34 \pm 0.51)$ than for nonhorizontal motion angles $[\mathrm{M}=0.65 \pm 0.23 ; F(1,3)=16.7$, $P=0.007]$. However, main effects did not depend on motion angles: We replicated the performance benefit for pursuit regardless of motion angle and showed a significant difference in motion prediction performance $\left(\mathrm{d}^{\prime}\right)$ between pursuit and fixation trials $[F(1,6)=7.9, P=0.03]$. As in experiments 1 and 2 , regardless of motion angle, perceptual performance, reflected both in d' (Fig. 8, A-C) and proportion correct (Fig. 8, $D-F$ ), increased with increasing presentation duration $[F(2,12)=17.7$, $P=0.0003]$. However, motion prediction did not depend on trajectory length $[F(2,12)=3.2, P=0.08]$, although Fig. 8 indicates a trend for performance in pursuit trials with longer presentation duration to increase with increasing trajectory length. None of the tested interactions were significant. These results imply that the ability to predict where a moving object is going depends more on temporal (the time to reach a decision) than spatial parameters (the distance traveled), at least for the spatiotemporal conditions tested here.

\section{DISCUSSION}

The present study provides direct evidence for a beneficial effect of smooth pursuit eye movements on the perception of motion direction. In three experiments, we showed that the prediction of a moving target's trajectory was better during pursuit than during fixation. This effect was independent of the retinal motion signal: pursuit produced better perceptual performance than fixation when retinal stimulation was similar (experiment 1) and when it was different (experiment 2). Further, the effect scaled with presentation duration and distance between ball and goal, but not with trajectory length (experiment 3), indicating the importance of temporal rather than spatial aspects of target motion.

\section{Contribution of Internal and External Motion Signals to the Performance Benefit during Pursuit}

What is the reason for the performance benefit during pursuit? In "eye soccer," the observers' task was to estimate the physical motion direction of the ball and the goal in two different situations, during fixation and during pursuit. In both cases, the physical motion of the targets at any instant of time, relative to the head/body (e.g., quantified in degrees per second), is simply

$$
\begin{aligned}
& M_{B}=R_{B}+E \text { (for the ball) } \\
& M_{G}=R_{G}+E \text { (for the goal) }
\end{aligned}
$$

where $R_{B}$ and $R_{G}$ are the retinal velocities of the ball and goal, with $\left(\mathrm{R}_{\mathrm{B}-}\right.$ hat $)$ and $\left(\mathrm{R}_{\mathrm{G}-\text { hat }}\right.$ ) being their estimates, respectively. $\mathrm{E}$ is the velocity of the eye and $\mathrm{E}$.hat its estimate. Note that estimates are imprecise and include random and nonrandom errors (i.e., noise and bias, respectively). The motion processing system in the brain has to estimate $\mathrm{M}_{\mathrm{B}}$ and $\mathrm{M}_{\mathrm{G}}$ from these noisy sources. During fixation, the eyes are more or less stationary; during pursuit, both eyes smoothly rotate in their orbits. In both cases, the motion estimate is based on a combination of retinal and extraretinal velocity estimates, but 

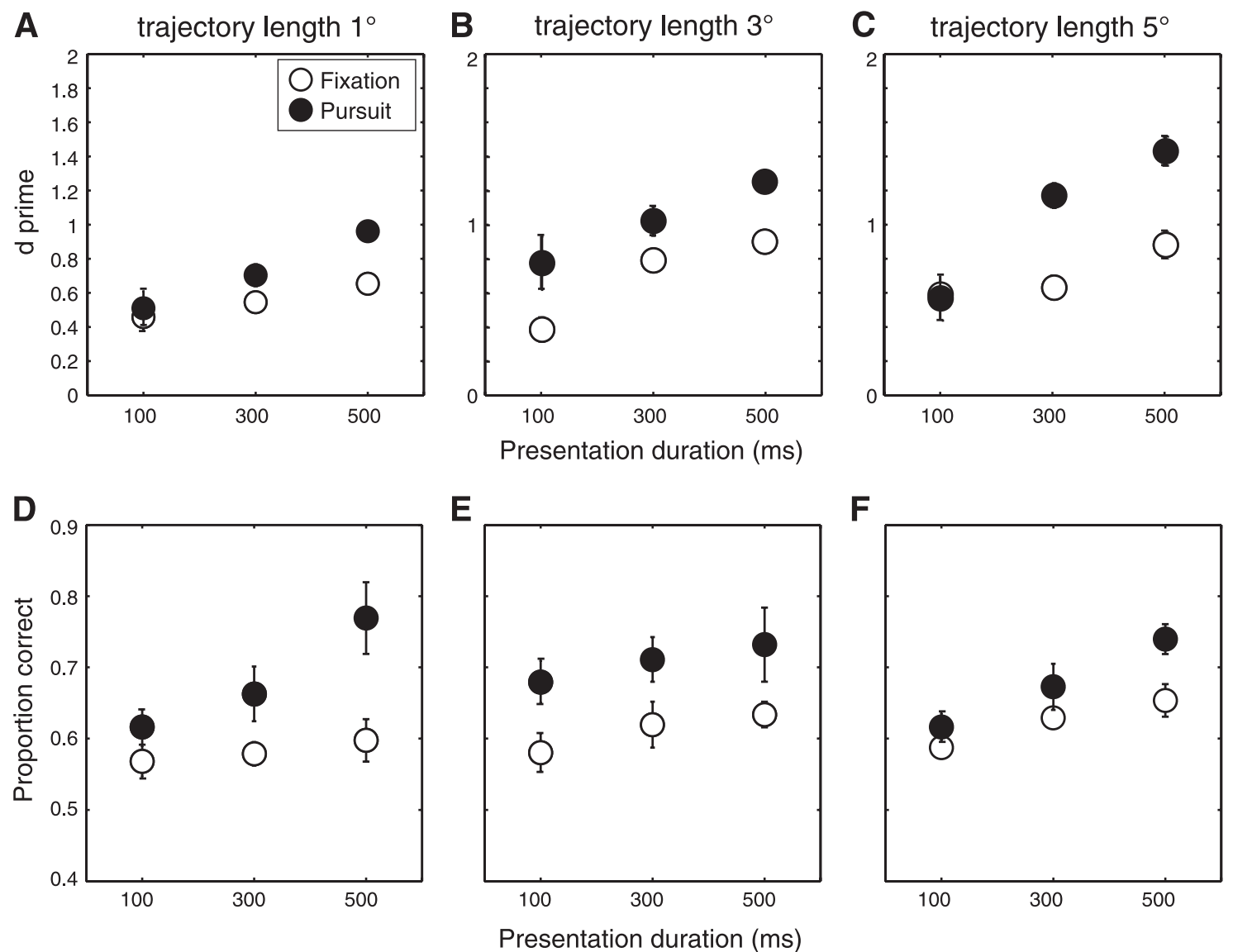

Fig. 8. Comparison of perceptual performance ( $\mathrm{d}^{\prime}$ and $\mathrm{PC}$ ) between pursuit (black) and fixation trials (white) in seven observers. $A$ : trajectory length $1^{\circ}$. $B: 3^{\circ}$. C: $5^{\circ} . D-F:$ proportion correct. Data are means \pm SE.

the values assigned to these estimates differ (see Table 2). Recall that we tested two different fixation conditions, one in which observers were instructed to fixate on a stationary ball while the goal moved towards the ball (experiment 1), and another in which observers were instructed to fixate on a stationary goal while the ball moved towards the goal (experiment 2). In both experiments, we found a performance benefit during pursuit, although retinal velocity estimates of ball and goal were similar during pursuit and fixation in experiment 1 and different in experiment 2. During both pursuit and fixation, $\mathrm{R}_{\mathrm{B}-}$ hat and $\mathrm{R}_{\mathrm{G}-\text { hat }}$ have to be estimated from the retinal slip. Our data show that performance during pursuit and fixation was better if retinal slip was lower (Fig. 5) and that retinal slip was higher during pursuit than during fixation (Table 1). It is therefore unlikely that differences in the estimation of $\mathrm{R}$ were responsible for the performance difference between pursuit and fixation.

Table 2. Value assumptions for estimates of retinal ball and goal velocity signals and extraretinal velocity signals during pursuit and fixation in experiments 1 and 2

\begin{tabular}{llll}
\hline \hline & \multicolumn{1}{c}{ E_hat } & \multicolumn{1}{c}{$\mathrm{R}_{\mathrm{B} \_ \text {hat }}$} & \multicolumn{1}{c}{$\mathrm{R}_{\mathrm{G} \_ \text {hat }}$} \\
\hline Pursuit & Nonzero value & Approx. 0 & Nonzero value \\
Fixation (experiment 1) & Approx. 0 & Approx. 0 & Nonzero value \\
Fixation (experiment 2) & Approx. 0 & Nonzero value & Approx. 0 \\
\hline
\end{tabular}

E_hat, estimate of eye velocity; $\mathrm{R}_{\mathrm{B} \_}$hat and $\mathrm{R}_{\mathrm{G} \_}$hat, estimates of the retinal velocities of the ball and goal, respectively.
How could the estimation of E contribute to the performance benefit in pursuit? E can be estimated from three different signals: 1) a retinal signal from physically stationary objects; 2) an afferent, proprioceptive signal from the eye muscles (Sherrington 1918); and 3) an "efference copy" or "corollary discharge" of the oculomotor command (von Helmholtz 1910/ 1962). Afferent and efferent signals are used by the motor system for calibration and online control of eye movements [for a review, see Sommer and Wurtz (2008)], and they can also inform perception (e.g., Gauthier et al. 1990; Stark and Bridgeman 1983). We hypothesize that E_hat may be computed from different sources during pursuit and fixation and that this difference might account for the performance difference. Whereas pursuit eye velocity may be compensated by extraretinal signals, for instance, to reduce perceived motion smear (Tong et al. 2008), velocity compensation during fixational eye movements seems to rely on retinal signals alone (Poletti et al. 2010). Our paradigm contained no visual references aside from the ball and the goal, presumably making a possible retinal-motion-based estimation of $\mathrm{E}$ during fixation imprecise. In addition, pursuit might recruit more attentional resources than fixation, resulting in better motion perception during pursuit. It is well documented that visual spatial attention is closely linked to the pursuit target [e.g., Lovejoy et al. (2009)] but can, at the same time, be flexibly allocated to other locations (Heinen et al. 2011). In "eye soccer," this might result in perceptual performance benefits with regard to both ball and goal location. 
Neurophysiological Basis for Motion Prediction During Pursuit

Our data indicate that the perceptual system might use an eye-motion signal, generated internally by the oculomotor system, to derive a better prediction of motion direction during pursuit than during fixation. Where do these signals originate? Generally, internal signals are either used to enable 1) better motor performance, i.e., more accurate eye movements, or, as in our study; and 2) better perceptual judgments.

1) Internal motion signals can be used for the execution of pursuit itself. Ongoing pursuit can be reasonably well maintained at a lower gain in the absence of a visual target. The predictive pursuit, predictive maintenance, and predictive recovery have been studied in experimental paradigms in which a moving target was transiently occluded (Becker and Fuchs 1985; Bennett and Barnes 2003-2006; Boman and Hotson 1992; Mrotek and Soechting 2007b; Orban de Xivry et al. 2006). It seems to rely on a combination of reflexive and voluntary control mechanisms. An eye-velocity memory that is continuously updated by efference-copy signals could be responsible for the maintenance of pursuit during an occlusion, while predictive recovery and scaling to the reappearing target's velocity changes have to be under voluntary control through extraretinal signals (Barnes and Asselman 1991; Bennett and Barnes 2004, 2006).

Neurophysiological studies on predictive pursuit show that these responses are mediated by activity in the supplementary eye field (SEF), a region in the dorsomedial frontal cortex (de Hemptinne et al. 2007, 2008; Heinen and Liu 1997; Missal and Heinen 2004). Heinen and colleagues (Kim et al. 2005) developed a paradigm to test the temporal dynamics of SEF activity related to cognitive expectations about target motion. In "ocular baseball," monkeys had to make or withhold a pursuit eye movement to a moving target, based on a simple rule. While fixating in the center of a "strike zone," the monkey had to determine whether a target moving towards the strike zone would hit it (strike trials), in which case the target had to be tracked, or miss it (ball trials), in which case fixation had to be maintained. Recordings in the SEF during the task revealed two types of neurons, reflecting target motion prediction and movement execution.

2) Internal motion signals can also affect perceptual judgments. Studies on predictive pursuit are mostly about prediction of more or less constant stimulus motion across several trials and the effect of predictive motion signals on pursuit characteristics. In contrast, our study is concerned with the extrapolation of stimulus motion within one trial and the effect of pursuit on perception. A recent study suggests that SEF neurons might not only be involved in target motion prediction for pursuit but also for perception (Shichinohe et al. 2009). This premotor area might therefore be a possible source of the related internal motion signal in the current study.

\section{What Does Perception “Know” About Pursuit?}

Some previous studies have demonstrated that concurrent eye or hand tracking can benefit perception but did not directly compare perception and pursuit. Wexler and Klam (2001) showed that a moving object appears to be positioned further back along its trajectory during pursuit than during fixation. However, if observers were actively moving the target with their hand, engaging in pursuit led to a more veridical position estimate. Unfortunately, the authors measured eye movements in separate, reduced versions of the main experiments and did not directly compare pursuit and perceptual performance. Following a similar logic but using manual instead of eye tracking, Tanaka et al. (2009) found that target displacement estimation during an occlusion period was more precise during manual tracking than during passive viewing. Eye movements were not measured in this study.

With regard to other perceptual tasks, many studies have indicated that the direction or speed of either the pursuit target or a secondary target can be altered by pursuit, relative to the physical motion of the target or to how the target appears to move during fixation. Most of these studies dealt with motion speed (Aubert 1887; Filehne 1922; Freeman and Banks 1998; Haarmeier and Thier 1998; von Fleischl 1882; Wertheim and Van Gelder 1990). The few studies on motion direction found that the direction of a secondary target was misperceived during pursuit, presumably because the eye speed was underestimated (Festinger et al. 1976; Morvan and Wexler 2009; Souman et al. 2005). However, an underestimation of eye speed will not affect the perceived direction of the eye movement target and is therefore unlikely to affect performance in our task. For the perceived direction of the pursuit target, Krukowski et al. (2003) found no difference between pursuit and fixation in a perceptual direction discrimination task. Direction thresholds were similar during fixation and pursuit, and perceptual performance was not related to pursuit gain. These findings are difficult to compare to ours, as these authors used a memory task with two intervals in which a visual motion signal had to be compared with an internal reference. Such a memory task presumably involves more processing stages and may be more difficult than our motion prediction task so that a possible pursuit benefit might have been masked.

We finally note that "eye soccer" is a laboratory experiment and not a computer animation of soccer; it does therefore not reflect the complexity of a real-world soccer game. In realworld soccer, more aspects of a ball's trajectory are uncertain than can be controlled in a reduced-cue environment, and players do not see the ball from a bird's eye perspective. Still, our findings might be relevant to situations where a sporting event (like a soccer match) is evaluated off the field (e.g., to analyze individual players' performance or to train soccer referees). While our findings might not have direct implications with regard to motor performance in soccer, they are relevant for the understanding of the effect of pursuit on perceptual performance, prerequisite for the development of experiments involving more natural stimuli (Rust and Movshon 2005).

\section{Conclusion}

Our results have two main theoretical implications: First, they show that the mere execution of a motor behavior can lead to a more precise estimate of motion direction than if it was based on retinal input alone. Second, it might be one of the main benefits of pursuit eye movements to provide information about a target's motion trajectory. The enhancement of spatial visual acuity is often mentioned as the main purpose of eye movements. Here, we show that in addition to enhancing visual acuity [e.g., Schütz et al. (2009)], pursuit can also enhance 
motion predictability. Better performance in perceptual tasks should lead to improved motor planning. To keep the eyes on a visual target, such as the ball in soccer, might therefore be a good strategy to improve perceptual as well as motor performance.

\section{ACKNOWLEDGMENTS}

We thank Jan-Christopher Werner for help with data collection.

\section{GRANTS}

This work was supported by the German Research Foundation (FOR 560 to M. Spering, A. C. Schütz, D. I. Braun, and K. R. Gegenfurtner and SP 1172/1-1 to M. Spering).

\section{DISCLOSURES}

No conflicts of interest, financial or otherwise, are declared by the author(s).

\section{REFERENCES}

Aubert H. Die Bewegungsempfindung [The sense of motion]. Pflügers Arch 40: 459-480, 1887.

Bahill AT, Baldwin DG, Venkateswaran J. Predicting a baseball's path. Am Sci 93: 218-225, 2006.

Bahill AT, LaRitz T. Why can't batters keep their eyes on the ball? Am Sci 72: 249-253, 1984.

Ball K, Sekuler R. A specific and enduring improvement in visual motion discrimination. Science 218: 697-698, 1982.

Barnes GR, Asselman PT. The mechanism of prediction in human smooth pursuit eye movements. J Physiol 439: 439-461, 1991.

Barnes GR, Collins CJS. The influence of briefly presented randomized target motion on the extraretinal component of ocular pursuit. J Neurophysiol 99: 831-842, 2008.

Becker W, Fuchs AF. Prediction in the oculomotor system: smooth pursuit during transient disappearance of a visual target. Exp Brain Res 57: 562575, 1985.

Bennett SJ, Barnes GR. Human ocular pursuit during the transient disappearance of a visual target. J Neurophysiol 90: 2504-2520, 2003.

Bennett SJ, Barnes GR. Predictive smooth ocular pursuit during the transient disappearance of a visual target. J Neurophysiol 92: 578-590, 2004.

Bennett SJ, Barnes GR. Timing the anticipatory recovery in smooth ocular pursuit during the transient disappearance of a visual target. Exp Brain Res 163: 198-203, 2005.

Bennett SJ, Barnes GR. Smooth ocular pursuit during the transient disappearance of an accelerating visual target: the role of reflexive and voluntary control. Exp Brain Res 175: 1-10, 2006.

Boman DK, Hotson JR. Predictive smooth pursuit eye movements near abrupt changes in motion direction. Vision Res 32: 675-689, 1992.

Bradley DC, Maxwell M, Andersen RA, Banks MS, Shenoy KV. Mechanisms of heading perception in primate visual cortex. Science 273: $1544-$ 1547, 1996.

Brenner E, Smeets JB. Continuous visual control of interception. Hum Mov Sci, in press: doi:10.1016/j.humov.2010.12.007, 2011.

Brenner E, Smeets JB. Sources of variability in interceptive movements. Exp Brain Res 195: 117-133, 2009.

Chukoskie L, Movshon JA. Modulation of visual signals in macaque MT and MST neurons during pursuit eye movement. J Neurophysiol 102: 32253233, 2009.

Collewijn H, Tamminga EP. Human smooth pursuit and saccadic eye movements during voluntary pursuit of different target motions on different backgrounds. J Physiol 351: 217-250.

de Hemptinne C, Lefèvre $\mathbf{P}$, Missal $\mathbf{M}$. Neuronal basis of directional expectation and anticipatory pursuit. J Neurosci 28: 4298-4310, 2008.

de Hemptinne C, Nozaradan S, Duvivier Q, Lefèvre P, Missal M. How do primates anticipate uncertain future events? J Neurosci 27: 4334-4341, 2007.

Dicke PW, Chakraborty S, Thier P. Neuronal correlates of perceptual stability during eye movements. Eur J Neurosci 27: 991-1002, 2008.

Festinger L, Sedgwick HA, Holtzman JD. Visual perception during smooth pursuit eye movements. Vision Res 16: 1377-1386, 1976.
Filehne W. Über das optische Wahrnehmen von Bewegungen (On the optical perception of movement). Z Sinnesphysiol 53: 134-145, 1922.

Freeman TC, Banks MS. Perceived head-centric speed is affected by both extra-retinal and retinal errors. Vision Res 38: 941-945, 1998.

Furmanski CS, Engel SA. An oblique effect in human primary visual cortex. Nat Neurosci 3: 535-536, 2000.

Gauthier GM, Nommay D, Vercher JL. The role of ocular muscle proprioception in visual localization of targets. Science 249: 58-61, 1990.

Gegenfurtner KR, Franz VH. A comparison of localization judgments and pointing precision. $J$ Vis 7: 11.158-12, 2007.

Gibson JJ, Smith OW, Steinschneider A, Johnson CW. The relative accuracy of visual perception of motion during fixation and pursuit. Am J Psychol 70: 64-68, 1957.

Haarmeier T, Thier P. An electrophysiological correlate of visual motion awareness in man. J Cogn Neurosci 10: 464-471, 1998.

Heinen SJ, Liu M. Single-neuron activity in the dorsomedial frontal cortex during smooth-pursuit eye movements to predictable target motion. Vis Neurosci 14: 853-865, 1997.

Heinen SJ, Jin Z, Watamaniuk SNJ. Flexibility of foveal attention during pursuit. J Vis 11: 1-12, 2011.

Inaba N, Shinomoto S, Yamane S, Takemura A, Kawano K. MST neurons code for visual motion in space independent of pursuit eye movements. $J$ Neurophysiol 97: 3473-3483, 2007.

Keller EL, Khan NS. Smooth-pursuit initiation in the presence of a textured background in monkey. Vision Res 26: 943-955, 1986.

Kim YG, Badler JB, Heinen SJ. Trajectory interpretation by supplementary eye field neurons during ocular baseball. J Neurophysiol 94: 1385-1391, 2005.

Krukowski AE, Pirog KA, Beutter BB, Brooks KR, Stone LS. Human discrimination of visual direction of motion with and without smooth pursuit eye movements. $J$ Vis 3: 831-840, 2003.

Land MF. Eye movements and the control of actions in everyday life. Prog Retin Eye Res 25: 296-324, 2006.

Land MF, Furneaux S. The knowledge base of the oculomotor system. Philos $T$ R Soc B 352: 1231-1239, 1997.

Land MF, McLeod P. From eye movements to actions: how batsmen hit the ball. Nat Neurosci 3: 1340-1345, 2000.

Land MF, Tatler BW. Looking and Acting. Vision and Eye Movements in Natural Behaviou. Oxford, England: Oxford University Press, 2009.

Lee SM. Does your eye keep on the ball? The strategy of eye movement for volleyball defensive players during spike serve perception. Int J Appl Sports Sci 22: 128-137, 2010.

Lisberger SG, Morris EJ, Tychsen L. Visual motion processing and sensorymotor integration for smooth pursuit eye movements. Ann Rev Neurosci 10: 97-129, 1987.

Lovejoy LP, Fowler GA, Krauzlis RJ. Spatial allocation of attention during smooth pursuit eye movements. Vision Res 49: 1275-1285, 2009.

McKinney T, Chajka K, Hayhoe M. Pro-active gaze control in squash. J Vis 8: $111,2008$.

Missal M, Heinen SJ. Supplementary eye fields stimulation facilitates anticipatory pursuit. J Neurophysiol 92: 1257-1262, 2004.

Morvan C, Wexler M. The nonlinear structure of motion perception during smooth eye movements. $J$ Vis 9: 1-13, 2009.

Mrotek LA, Soechting JF. Target interception: hand-eye coordination and strategies. J Neurosci 27: 7297-7309, 2007a.

Mrotek LA, Soechting JF. Predicting curvilinear target motion through an occlusion. Exp Brain Res 178: 99-114, 2007b.

Orban de Xivry JJ, Bennett SJ, Lefevre P, Barnes GR. Evidence for synergy between saccades and smooth pursuit during transient target disappearance. J Neurophysiol 95: 418-427, 2006.

Osborne LC, Hohl SS, Bialek W, Lisberger SG. Time course of precision in smooth-pursuit eye movements of monkeys. J Neurosci 27: 2987-2998, 2007.

Poletti M, Listorti C, Rucci M. Stability of the visual world during eye drift. J Neurosci 30: 11143-11150, 2010.

Ripoll H, Bard C, Paillard J. Stabilization of head and eyes on target as a factor in successful basketball shooting. Hum Mov Sci 5: 47-58, 1986.

Rust NC, Movshon JA. In praise of artifice. Nat Neurosci 8: 1647-1650, 2005.

Schütz AC, Braun DI, Gegenfurtner KR. Object recognition during foveating eye movements. Vision Res 49: 2241-2253, 2009.

Shenoy KV, Bradley DC, Andersen RA. Influence on gaze rotation on the visual response of primate MSTd neurons. J Neurophysiol 81: 2764-2786, 1999. 
Sherrington CS. Observations on the sensual role of the proprioceptive nerve-supply of the extrinsic ocular muscles. Brain J Neurol 41: 332-342, 1918.

Shichinohe N, Akao T, Kurkin S, Fukushima J, Kaneko CRS, Fukushima $\mathbf{K}$. Memory and decision making in the frontal cortex during visual motion processing for smooth pursuit eye movements. Neuron 62: 717-732, 2009.

Soechting JF, Juveli JZ, Rao HM. Models for the extrapolation of target motion for manual interception. J Neurophysiol 102: 1491-1502, 2009.

Sommer MA, Wurtz RH. Visual perception and corollary discharge. Perception 37: 408-418, 2008.

Souman JL, Hooge IT, Wertheim AH. Perceived motion direction during smooth pursuit eye movements. Exp Brain Res 164: 376-386, 2005.

Stark L, Bridgeman B. Role of corollary discharge in space constancy. Percept Psychophys 34: 371-380, 1983.

Stone LS, Krauzlis RJ. Shared motion signals for human perceptual decisions and oculomotor actions. $J$ Vis 3: 725-736, 2003.

Tanaka H, Worringham C, Kerr G. Contributions of vision-proprioception interactions to the estimation of time-varying hand and target locations. Exp Brain Res 195: 371-382, 2009.

Tavassoli A, Ringach DL. When your eyes see more than you do. Curr Biol 20: 93-94, 2010.
Thier P, Haarmeier T, Chakraborty S, Lindner A, Tikhonov A. Cortical substrates of perceptual stability during eye movements. Neuroimage 14: 33-39, 2001.

Tong J, Stevenson SB, Bedell HE. Signals of eye-muscle proprioception modulate perceived motion smear. $J$ Vis 8: 1-6, 2008.

Turano KA, Heidenreich SM. Eye movements affect the perceived speed of visual motion. Vision Res 39: 1177-1187, 1999.

von Fleischl E. Physiologisch-optische notizen [Notes on physiological optics]. Sitzungsber Akad Wiss Wien 3: 7-25, 1882.

von Helmholtz H. Handbuch der Physiologischen Optik [Treatise on physiological optics]. New York: Dover, vol. 3, 1910/1962.

von Holst E, Mittelstaedt H. Das Reafferenzprinzip [The reafference principle]. Naturwissenschaften 37: 464-476, 1950.

Wertheim AH, Van Gelder P. An acceleration illusion caused by underestimation of stimulus velocity during pursuit eye movements: Aubert-Fleischl revisited. Perception 19: 471-482, 1990.

Wexler M, Klam F. Movement prediction and movement production. J Exp Psychol Hum Percept Perform 27: 48-64, 2001.

Whitaker D, Levi DM, Kennedy GJ. Integration across time determines path deviation discrimination for moving objects. PLos One 3: e1930, 2008 .

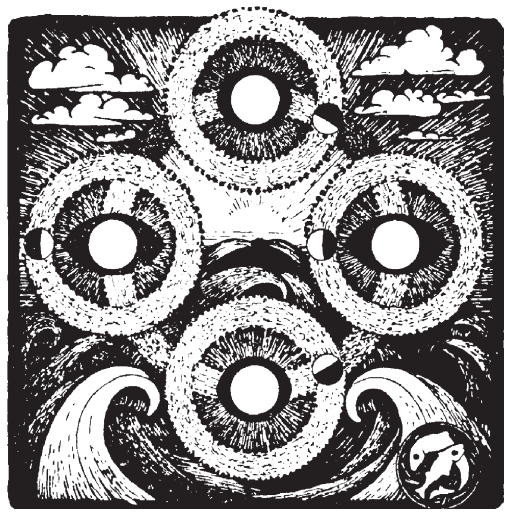

